\title{
HEALTH-RELATED PHYSICAL FITNESS AND ASSOCIATED SOCIODEMOGRAPHIC FACTORS IN ADOLESCENTS FROM A BRAZILIAN STATE CAPITAL
}

\author{
EDIO LUIZ PETROSKI ${ }^{1,4}$ *, DIEGO AUGUSTO SANTOS SILVA ${ }^{1}$, \\ JOÃO MARCOS FERREIRA DE LIMA E SILVA ${ }^{2}$, ANDREIA PELEGRINI ${ }^{3}$ \\ ${ }^{1}$ Federal University of Santa Catarina, Post-Graduate Program in Physical Education, Sport Center, Florianópolis, SC. Brazil \\ ${ }^{2}$ Federal Institute of Education, Science and Technology of Ceará, Campus North Juazeiro, CE, Brazil \\ ${ }^{3}$ State University of Santa Catarina, Florianópolis, SC. Brazil \\ ${ }^{4}$ CAPES Foundation - grant BEX 0951/10-2
}

\begin{abstract}
Purpose. The aim of this study was to investigate the associations between health-related physical fitness and sociodemographic factors in students from a capital city of a Brazilian state. Methods. A cross-sectional study was conducted on 605 students aged 15 to 19 years. Sociodemographic data were collected, including gender, age, education level and family income, and correlated to physical fitness levels. Results. The percentages of students with unhealthy body composition, unhealthy skeletal muscle fitness and aerobic fitness levels were $23.8 \%, 34.4 \%$ and $30.5 \%$, respectively. There was a trend for fewer male adolescents (OR: 0.65; IC95\%: 0.42-0.98) to have unhealthy body composition. Students from lower socioeconomic families were less likely to have musculoskeletal unfitness (OR $=0.60$; IC95\%: 0.41-0.89). In relation to aerobic fitness, male students $(\mathrm{OR}=3.86$; IC95\%: 2.67-5.58) and those aged 17-19 years ( $\mathrm{RO}=1.49$; IC95\%: 1.02-2.177) were more likely to be unfit. Conclusions. It is important to encourage young people to take part in sports and physical activities at moderate to vigorous intensities in order to improve their body composition, aerobic capacity and physical fitness.
\end{abstract}

Key words: physical fitness, physical exertion, students, health, motor activity, cross-sectional study, schoolchildren

\section{Introduction}

Studies show that low levels of health-related physical fitness (HRPF) during adolescence are associated with an increased risk of developing chronic degenerative diseases and a higher mortality risk in adulthood [1]. One way of preventing these negative outcomes is to regularly engage in a sufficient level of physical activity [2]. Despite this, research conducted in schools reports elevated prevalence rates of little to no physical activity among adolescents [3-5].

Health-related physical fitness has been defined as the capacity to take part in daily physical activities without overexertion in combination with demonstrating a number of characteristics and abilities that are associated with low risk of developing hypokinetic diseases [6]. Therefore, an excellent level of HRPF does not demand extreme levels of physical fitness nor does it require individuals to perform similarly to athletes, who generally perform exceptionally well in a range of physical tests. The components of HRPF can be maintained at healthy levels through physical exercise directed at improving aerobic resistance, muscle strength, muscle resistance and flexibility and lowering overall body fat levels.

\footnotetext{
* Corresponding author.
}

Motor test batteries used to assess the HRPF or athletic fitness of children and adolescents are created by combining several physical tests into one. Physical fitness batteries are based either on standards of normality or on selected health criteria. Those that are based on standards are compiled by testing a representative population sample, with cut-offs then established in the form of percentiles. Subjects that fall above or below these percentiles are defined as having healthy or unhealthy levels of physical fitness.

Physical fitness batteries that are based on health criteria offer the advantage that they identify physical fitness levels that may expose a given person to certain health risks. Lunardi and Petroski [7], for example, investigated 11-year-old adolescents and found that elevated values of body composition (i.e. a body mass index $[\mathrm{BMI}] \geq 19.3 \mathrm{~kg} / \mathrm{m}^{2}$ ) could be used as a diagnostic criterion for an unhealthy lipid profile, predicting elevated triglycerides among females and elevated total cholesterol and LDL-cholesterol in males.

A large number of different factors have an influence on HRPF, among the most important being sociodemographic factors. For example, males have been shown to have better fitness levels than females [8]. Santos et al. [9] found that the likelihood of a girl having healthy HRPF, on the basis of the four HRPF tests taken from the Fitnessgram battery, is approximately $50 \%$ of that for a boy. Luguetti et al. [10] studied schoolchildren aged 7 to 16 and observed that the proportion of them with 
unhealthy HRPF was elevated at all ages and was more common among girls.

Age and school year are also factors related to HRPF. In Portugal, Lopes et al. [8] observed a tendency for HRPF to reduce as chronological age increased. Another sociodemographic factor that appears to modulate physical fitness is economic status. Although still the subject of debate, adolescents from families with upper socioeconomic status have more opportunities to take part in physical activities and sports at gyms and sports clubs and to engage in adventure activities because of their increased ability to acquire sports equipment and materials [11]. In contrast, although Dumith et al. [12] did find a high prevalence of low physical fitness in a sample of Brazilian schoolchildren, they did not find any link with economic status.

With reference to the literature on the subject, it can be observed that certain issues related to the health-related physical fitness of adolescents have not yet received sufficient research attention. These include questions such as whether males and females differ in terms of the components of health-related physical fitness, whether physical fitness is affected by an increase in age during adolescence and whether there is a relationship between the components of fitness and socioeconomic and demographic factors.

It is therefore evident that there is a need to investigate HRPF and the factors related to it in adolescents, with an objective of identifying at-risk individuals early enough in order to successfully intervene and change their lifestyle while they are still adolescents. This is primarily because adolescence is a critical period when unhealthy behaviours and habits can be easily acquired and continue to persist into adulthood.

Few studies have yet been undertaken in secondary education to analyse HRPF and the factors associated with it. The objective of this study is therefore to verify associations between HRPF and sociodemographic factors in adolescents from a Brazilian state capital. The hypothesis being tested in this study is that sociodemographic factors determine components of HRPF in Brazilian adolescents.

\section{Material and methods}

The study was approved by the Human Research Ethics Committee at the Universidade Federal de Santa Catarina (Protocol number 372/2006). This was a descriptive cross-sectional study conducted during the second half of 2007 in the city of Florianópolis, which is the state capital of Santa Catarina and is located in southern Brazil. Brazil has 26 state capitals, one for each of the 26 states in Brazil, plus the Distrito Federal in which the Federal capital Brasilia is located. The state capitals are urban centres and have the largest population concentrations in their respective states. The capitals also host the state governments, financial centres and major industries and are responsible for a large proportion of the states' economies.

Sample selection was two-stage, first stratified by geographic region and then clustered by school classes. Part of the city of Florianópolis is located on the South American mainland and part is on the island of Santa Catarina. In stage one, the city was divided into five geographical regions as: city centre, continental region, east of the island, north of the island and south of the island. The largest school in each region was selected and then classes from that school were chosen by lots until a sample representative of the geographic area had been selected. In stage two, all adolescents in the chosen classes who attended school on the day of data collection were invited to take part.

Several different sample sizes were calculated as this study was part of a larger project investigating a range of different health-related outcomes. The sample used for the analysis described here was calculated to study the prevalence of physical unfitness. The calculation procedure for finite populations was used to find the minimum sample size for an estimated outcome prevalence of $60 \%$ [13], an acceptable error of five percentage points, a 95\% confidence level and a design effect of 1.5 , and then the result was increased by $10 \%$ to account for possible refusals and losses from the sample. On this basis, a minimum of 591 schoolchildren were needed. The characteristics of the sample process, which stipulated the inclusion of all individuals in each chosen cluster, meant that the actual sample comprised 892 adolescents.

Eligibility criteria for participation in this investigation were as follows: enrolment in the state education system, attendance on the day of data collection and being between ages 15 to 19. The upper limit was based on the World Health Organization [14] definition of the end of adolescence and the subjects' educational context (they were all attending secondary education). The exclusion criteria were: (a) age greater than 19 years; (b) pregnancy; (c) failure to provide a free and informed consent form signed either by their parents or guardians, in the case of minors, or by the adolescents themselves if over the age of 18. Students were defined as refusals if they did not wish to take part in the study or as sample losses if they did not answer the entire questionnaire.

Subjects were given a minimum of 3 days' notice prior to data collection. On the day on which they were informed of the study, they were also provided the free and informed consent forms and the test procedures were explained to them. The tests were aimed at being administered during physical education classes, although this was not always possible.

Socioeconomic status was classified using a questionnaire produced by the Brazilian Association of Market Research Companies (ABEP - Associação Brasileira de Empresas de Pesquisa, 2003) [15]. In this questionnaire 
the answers are converted into scores using a points system and the results divide the Brazilian population into economic classes according to their spending power. The ABEP criteria result in five classes, from "A" to "E", in descending order of spending power. The questionnaire covers the head of the family's educational level, the number of domestic servants the family employs and nine items related to consumer durables that the family possesses. After preliminary analysis of frequency distributions, for the purposes of this study, socioeconomic status was collapsed down to three classes: high "A"; medium "B" and low "C+D+E".

The components of HRPF were measured using tests, instruments and standards recommended by the Canadian Physical Activity, Fitness \& Lifestyle Approach Protocol [16], which is based on health-related criteria. The entire examination analysed three main components, with the first being body composition in terms of (a) body mass index (BMI), (b) waist circumference (WC) and (c) the sum of five skin folds $-\Sigma 5 \mathrm{Sk}$ (triceps, biceps, subscapular, iliac crest and medial calf).

The second measured component was skeletal muscle fitness in terms of (a) flexibility - by a modified "sit-toreach" test, (b) handgrip strength test - using a dynamometer to measure each hand alternately, with two attempts per hand (the best score for each hand were added together to provide a global score), (c) lower limb strength - the best score from three attempts at the standing jump test (with the following formula used to calculate the final result: Lower Limb Strength $(W)$ $=[60.7 \times$ height of jump $(\mathrm{cm})]+[45.3 \times$ body weight $(\mathrm{kg})]$ $-2055)$, (d) press ups - continued up to exhaustion, with males supporting themselves with their toes and females supporting themselves with their knees, (e) partial situps - (for one-minute) the speed of this test was set by a metronome adjusted to 50 beats per minute, and (f) back extension - the back was extended and the trunk supported for 3 minutes.

The final third component was aerobic fitness, measured by a (a) bench test, performed using a stepped ergometer which the subject had to ascend and descend at the speed demanded by the test protocol, indicated by a metronome. The test returned a score for aerobic fitness according to the following equation: aerobic fitness score $=10 \times\left[17.2+\left(1.29 \times \mathrm{O}_{2}\right.\right.$ consumption $)-$ $(0.09 \times$ body weight in $\mathrm{kg})-(0.18 \times$ age in years $)$.

Scores for the components of the body composition domain (BMI, WC and $55 \mathrm{Sk}$ ), the skeletal muscle fitness domain (flexibility, muscle strength/resistance) and the aerobic fitness domain were generated from the combined results of each test [16]. Each component was then classified into one of five categories based on the scores' implications for health: (1) "must improve", (2) "regular", (3) "good", (4) "very good" or (5) "excellent". For the purposes of this study, the five HRPF categories were collapsed down to "healthy" (categories 3, 4 and 5) or "unhealthy" (categories 1 and 2).
Fieldwork was carried out by a team of four professors and postgraduates studying for master's degrees and doctorates in Physical Education. These assessors were trained in all of the procedures in advance in order to standardize data collection. Each team member was made responsible for data collection related to one test in order to avoid interobserver variability, thereby increasing the reliability of data. Furthermore, a pilot study was conducted at a school that was not part of the definitive sample in order to test the used instruments and general logistics in the field. Anthropometric measurements were taken by anthropometrists who had been trained and certified by the International Society for the Advancement of Kinanthropometry (ISAK). All of them had a Technical Error of Measurement within acceptable limits for anthropometric measurements [16].

Descriptive statistics were used to analyse the data. Both crude and adjusted binary logistic regression models were used to test for associations between physical unfitness (the dependent variable) and sociodemographic variables. The adjusted model was constructed using the stepwise method, based on likelihood ratios, to calculate adjusted odds ratios (with 95\% confidence intervals), starting from a model including those variables which had a $p$ value of 0.20 or less in the crude analysis. Statistical analysis was performed using SPSS statistical analysis software (SPSS ver. 15.0, IBM, USA) to calculate odds ratios and their respective $95 \%$ confidence intervals.

\section{Results}

From the total number of adolescents in class on the day of data collection, $21.74 \%$ refused to take the physical tests, $8.41 \%$ were considered sample losses and $2 \%$ were excluded because they were outside the age range. The final sample taken under consideration comprised of 605 secondary education students with a mean age of 16.1 years (standard deviation $=1.0$ ).

It can be observed from Table 1 that the majority of the sample were female, aged 15 to 16 , were in the eleventh grade and had middle socioeconomic status. Table 1 illustrates the prevalence rates of adolescents with unhealthy HRPF levels. In this sample, $23.8 \%$ (95\%CI: 20.3-27.2) were unhealthy in terms of body composition, $34.4 \%$ (95\%CI: 30.5-38.1) were unhealthy for skeletal muscle fitness and 30.5\% (95\%CI: 26.8-34.2) were unhealthy in terms of their aerobic fitness. Table 1 lists the prevalence of adolescents who did not meet the recommended level of each component of health-related physical fitness for each of the analysed sociodemographic variables.

Crude logistic regression analysis indicated that male adolescents and twelfth-grade students were less likely to have unhealthy body composition. For skeletal muscle fitness, the subsets least likely to be unhealthy were in the twelfth grade and had lower economic sta- 


\section{HUMAN MOVEMENT}

E.L. Petroski et al., Health and physical fitness in adolescents

Table 1. Distribution of the sample by sociodemographic variables and prevalence of failure to achieve the levels recommended for good health in the components of health-related physical fitness, broken down by the sociodemographic variables

\begin{tabular}{|c|c|c|c|c|}
\hline & \multirow{2}{*}{$\begin{array}{c}\text { Sample } \\
\text { distribution }\end{array}$} & \multicolumn{3}{|c|}{ Health-related physical fitness: Unhealthy } \\
\hline & & body composition & skeletal muscle fitness & aerobic fitness \\
\hline & $N(\%)$ & $\%(95 \% \mathrm{CI})$ & $\%(95 \% \mathrm{CI})$ & $\%(95 \% \mathrm{CI})$ \\
\hline Total & 605 & $23.8(20.3-27.2)$ & $34.4(30.5-38.1)$ & $30.5(26.8-34.2)$ \\
\hline \multicolumn{5}{|l|}{ GENDER } \\
\hline Female & $388(64.1)$ & $26.5(22.1-30.9)$ & $33.8(29.0-38.4)$ & $20.1(16.0-24.1)$ \\
\hline Male & $217(35.9)$ & $18.8(13.6-24.1)$ & $35.4(29.1-41.9)$ & $49.3(42.5-56.1)$ \\
\hline \multicolumn{5}{|c|}{ AGE (YEARS) } \\
\hline $15-16$ & $383(63.3)$ & $24.8(20.4-29.1)$ & $36.2(31.4-41.1)$ & $27.2(22.7-31.7)$ \\
\hline 17-19 & $222(36.7)$ & $22.1(16.6-27.5)$ & $31.1(24.9-37.2)$ & $36.1(29.6-42.4)$ \\
\hline \multicolumn{5}{|l|}{ GRADE } \\
\hline $10^{\text {th }}$ & $180(29.8)$ & $22.7(16.5-28.9)$ & $33.8(26.9-40.8)$ & $29.2(22.4-35.9)$ \\
\hline $11^{\text {th }}$ & $259(42.8)$ & $30.5(24.8-36.1)$ & $38.6(32.6-44.5)$ & $28.2(22.6-33.7$ \\
\hline $12^{\text {th }}$ & $166(27.4)$ & $14.4(9.1-19.8)$ & $28.3(21.3-35.2)$ & $35.3(27.9-42.7)$ \\
\hline \multicolumn{5}{|c|}{ SOCIOECONOMIC STATUS } \\
\hline Upper & $43(7.1)$ & $25.5(11.9-39.1)$ & $32.5(17.9-47.1)$ & $34.9(20.0-49.7)$ \\
\hline Middle & $374(61.8)$ & $22.4(18.2-26.7)$ & $38.2(33.2-43.1)$ & $32.3(27.5-37.1)$ \\
\hline Lower & $188(31.1)$ & $26.0(19.7-32.3)$ & $27.1(20.7-33.5)$ & $25.8(19.4-32.1)$ \\
\hline
\end{tabular}

95\%CI - confidence interval

Table 2. Crude logistic regression analysis for health-related physical fitness against the sociodemographic variables

\begin{tabular}{ccc}
\hline \multicolumn{3}{c}{ Health-related physical fitness: Unhealthy } \\
\hline body composition & skeletal muscle fitness & aerobic fitness \\
\hline OR $(95 \% \mathrm{CI})^{\dagger}$ & OR $(95 \% \mathrm{CI})^{\dagger}$ & OR $(95 \% \mathrm{CI})^{\dagger}$ \\
\hline
\end{tabular}

\begin{tabular}{llll}
\hline GENDER & & & \\
Female & 1 & 1 & 1 \\
Male & $0.64(0.42-0.96)^{* * *}$ & $1.08(0.76-1.53)$ & $3.88(2.69-.59)^{* * *}$ \\
\hline
\end{tabular}

\begin{tabular}{llll}
\hline AGE (YEARS) & 1 & 1 & 1 \\
$15-16$ & $0.86(0.58-1.27)$ & $0.79(0.56-1.13)^{* *}$ & $1.50(1.05-.15)^{* * *}$ \\
$17-19$ & & & $1.05(0.69-1.60)$ \\
\hline GRADE & $0.67(0.43-1.04)$ & $0.82(0.55-1.21)$ & 1 \\
$10^{\text {th }}$ & 1 & 1 & $1.39(0.91-2.12)$ \\
$11^{\text {th }}$ & $0.38(0.23-0.63)^{* *}$ & $0.63(0.41-.96)^{* * *}$ & $1.12(0.58-2.18)$ \\
$12^{\text {th }}$ & $1.18(0.57-2.45)$ & $0.78(0.40-1.53)$ & 1 \\
\hline SOCIOECONOMIC STATUS & 1 & 1 & $0.73(0.49-1.08)$ \\
Upper & $1.21(0.81-1.82)$ & $0.60(0.41-.88)^{* *}$ & 0.05 \\
Middle & Lower & & \\
\hline
\end{tabular}

OR - odds ratio; 95\%CI - confidence interval; ${ }^{\dagger}$ Crude logistic regression analysis; * $p \leq 0.20 ; * * p \leq 0.05$

tus. Male subjects were more likely to have poor aerobic fitness than females (Tab. 2).

The adjusted logistic regression model indicated that male adolescents (OR: 0.65; 95\% CI: 0.42-0.98) and twelfth-grade students (OR: 0.39; 95\% CI: 0.23-0.64) were less likely to have unhealthy body composition than females in the eleventh grade (Tab. 3). Adolescents with lower socioeconomic status were less likely (OR: 0.60; 95\% CI: 0.41-0.89) to have skeletal muscle unfitness, compared with those from middle-class families. With regard to aerobic resistance, male schoolchildren (OR: 3.86; 95\% CI: 2.67-5.58), and those aged 
Table 3. Adjusted logistic regression model for the health-related physical fitness components against the sociodemographic variables

\begin{tabular}{|c|c|c|c|}
\hline & \multicolumn{3}{|c|}{ Health-related physical fitness: Unhealthy } \\
\hline & body composition & skeletal muscle fitness & aerobic fitness \\
\hline & OR $(95 \% \mathrm{CI})^{\dagger}$ & OR $(95 \% \mathrm{CI})^{\dagger}$ & OR $(95 \% \mathrm{CI})^{\dagger}$ \\
\hline \multicolumn{4}{|l|}{ GENDER } \\
\hline Female & 1 & $\# \#$ & 1 \\
\hline Male & $0.65(0.42-0.98)^{* *}$ & & $3.86(2.67-5.58)^{* * *}$ \\
\hline \multicolumn{4}{|c|}{ AGE (YEARS) } \\
\hline $15-16$ & \#\# & 1 & 1 \\
\hline 17-19 & & $0.92(0.59-1.42)$ & $1.49(1.02-2.17)^{* * *}$ \\
\hline \multicolumn{4}{|l|}{ GRADE } \\
\hline $10^{\text {th }}$ & $0.69(0.44-1.07)$ & $0.81(0.54-1.22)$ & \#\# \\
\hline $11^{\text {th }}$ & 1 & 1 & \\
\hline $12^{\text {th }}$ & $0.39(0.23-0.64)^{* * *}$ & $0.66(0.41-1.47)$ & \\
\hline \multicolumn{4}{|c|}{ SOCIOECONOMIC STATUS } \\
\hline Upper & \#\# & $0.75(0.38-1.48)$ & \#\# \\
\hline Middle & & 1 & \\
\hline Lower & & $0.60(0.41-0.89)^{* *}$ & \\
\hline
\end{tabular}

OR - odds ratio; CI - confidence interval

$\dagger$ logistic regression model adjusted for those values that had a Wald test $p$ value $\leq 0.20$ in the crude analysis (Tab. 2 )

\#\# variables omitted from the multivariate model because their Wald test $p$ values were greater than 0.20

$* * p \leq 0.05$

17 to 19 years (OR: 1.49 ; 95\% CI: $1.02-2.17$ ) were more likely to have a low level of aerobic fitness than females and adolescents aged $15-16$ years.

\section{Discussion}

This study found worryingly high prevalence rates of low HRPF levels among adolescents. Additionally, male adolescents and those in the thirteenth-grade were less likely to have unhealthy body composition than females and those in the twelfth-grade. Furthermore, schoolchildren with lower socioeconomic status were less likely to have skeletal muscle unfitness than those with middle socioeconomic status. Male schoolchildren and older children were more likely to have low aerobic fitness than their female and younger peers.

Glaner [17], investigated HRPF in adolescents from urban and rural areas of the Brazilian states of Rio Grande do Sul and Santa Catarina and found a prevalence of unhealthy HRPF in the order of $90 \%$ for components of body composition and skeletal muscle fitness, and found that unfitness was more prevalent among urban dwellers, providing evidence of the influence the physical environment exerts on physical fitness. The present study was conducted in Florianopolis, which is the city with the highest Human Development Index of all the state capitals in Brazil. This is an urban area in which the behaviour of young people is becoming ever more sedentary, possibly combined with limited access to leisure facilities and adoption of a lifestyle with hypokinetic characteristics.

Body composition is considered to be part of HRPF and unhealthy body composition is associated with diseases such as obesity, diabetes and arterial hypertension [18]. Overweight adolescents have a greater probability of high blood pressure ( $\mathrm{PR}=1.95$ to 2.03 ) and hypertension $(\mathrm{PR}=4.22$ to 4.60$)$ with relation to their underweight/normal weight peers [19].

It is therefore apparent that public health promotion is in need of strategies that can raise adolescents' awareness and encourage them to strive for healthy body composition if health problems associated with obesity are to be avoided in adulthood. One way of combating and preventing these problems is by including additional programs at school, during physical education classes, as shown by Farias et al. [20]. These authors conducted a study in the city of Porto Velho, Roraima, Brazil, in which a program of guided physical activity was included in physical dducation classes for one academic year. The intervention group exhibited stable subscapular skin folds, BMI, body fat percentage and fat mass; and significant reductions in triceps skin folds and abdominal circumference. In contrast, the control group had increases in BMI, triceps skin folds, abdominal circumference and fat mass.

Skeletal muscle fitness was assessed using a series of physical tests making it possible to conduct a more detailed analysis of HRPF, which is one of the Canadian 
system's strong points. One in three of the adolescents studied here had low musculoskeletal physical fitness, which is commonly associated with functional limitations and an increased risk of sarcopenia and osteoporosis in later life. One prospective study investigated whether flexibility, strength, muscle resistance and physical activity predict bone and joint diseases and conditions. After 25 years of study, the authors concluded that adolescents who scored low in the skeletal component had a 96 times greater chance of lombalgia, postural problems and joint conditions than people with high scores [21].

The divergence between different motor test batteries can be observed by comparing the prevalence of skeletal muscle fitness found in this study with the prevalence rates reported by others. Ronque et al. [22] analysed the motor performance of upper socioeconomic status children from the Brazilian city of Londrina using the AAHPERD battery and found that more than $80 \%$ of the studied schoolchildren did not meet the minimum criteria for good health. The results of this study demonstrated that the adolescents with upper socioeconomic status had higher scores for the skeletal muscle component than those with lower socioeconomic status. Studies in literature that have investigated upper status [22] and lower status adolescents [23] reported that the prevalence of low scores was higher among lower socioeconomic status children.

Another important component of HRPF that has a significant impact on individuals' health is aerobic fitness. The high prevalence of adolescents with low levels of aerobic fitness observed in this study is of great concern because poor aerobic fitness is associated with lipid profile imbalances, metabolic disorders and an increased risk of obesity. After a 9-month case-control study of schoolchildren from Madison, Wisconsin (USA), it was shown that a school-based physical fitness program was capable of improving aerobic capacity. These results were associated with reductions in body fat and improvements in insulin resistance [24]. A school-based epidemiological study conducted in the south of Brazil observed that adolescents of both genders with low levels of aerobic fitness have a 4.10 times greater chance of abdominal obesity than their peers with healthy levels of aerobic fitness [25].

The present study found that gender was associated with aerobic fitness, indicating that male adolescents have an almost four times greater chance of poor aerobic fitness than females. Although comparison of aerobic fitness observations is complicated by the great diversity in methodologies and cut-off points employed by different studies, the study results are in line with the literature. Vasques et al. [26] used the Fitnessgram to investigate the aerobic fitness of adolescents and found that $68 \%$ of the boys and $37.8 \%$ of the girls did not reach the minimum levels recommended for good health. Similar results have been reported by Pelegrini et al. [27], who applied the AAHPERD criteria and observed low physical fitness levels, representing health risk in terms of cardiorespiratory fitness (boys: $80.8 \%$; girls: $77.6 \%$, $p<0.001)$. Notwithstanding, those results are in opposition to the results of other studies in the literature, which have indicated that males are fitter [21].

In the present study, adolescents aged 17 to $19 \mathrm{had}$ a 1.49 times lower chance of attaining a healthy score than the 15 to 16 age group. Malina [28] conducted an extensive systematic review to quantify secular changes in the aerobic fitness of children and adolescents. Among other results, they found that, according to 33 studies from 27 different countries, over the last 45 years the aerobic fitness of young people all over the world has been decreasing by $0.36 \%$ per year. Furthermore, it was also found that after puberty this loss intensifies as age increases, above all because of the inactive lifestyle that is ever more pervasive among adolescents.

Despite the scientific evidence illustrating the relationship between physical activity and health, young Brazilians are adopting ever more inactive or low-activity lifestyles. Silva et al. [3] conducted a survey in the capital city of a state in the northeast of Brazil and found that more than $75 \%$ of adolescents were inactive. Ceschini et al. [4] reported that $62.5 \%$ of the children attending secondary education in the city of São Paulo engaged in little or no physical activity. Another recent study reported that one in every four adolescents in a south administrative region of Brazil were inactive or underactive [5]. As was shown in a recent systematic review, estimates of this magnitude have been made in many different countries [2].

The present study did not compare relative and absolute oxygen consumption, but restricted the analysis to the proportion of a sample of schoolchildren who met the minimum criteria for good health, using lower cut-off points for females than for males [16]. Prospective studies that take direct assessments of aerobic fitness into account are needed before further conclusions can be drawn. In addition, this study does suffer from certain limitations. The sample was drawn exclusively from state-funded secondary schools. Therefore other sectors of the educational system, such as municipal schools, private schools and technical schools were not assessed. The conclusions arrived here are thus only related to the population studied. Another limitation is the number of children who refused to take the physical tests, which is evidence of a lack of motivation to exercise on the part of the participants and may have affected the results.

The findings reported in this study should serve as a starting point for future investigations conducted in other locations using the Canadian physical fitness battery, which includes a greater number of tests and measures for diagnosing HRPF levels and should therefore offer more specific results for these variables. Notwithstanding, further debate is needed in order to create 
intervention strategies aimed at encouraging school populations to engage in sufficient physical activity to maintain good health.

\section{Conclusion}

This study concluded that a low proportion of adolescent children in Florianopolis scored well for HRPF. Approximately one in every four children had an unhealthy body composition, and two in every three had low levels of aerobic and musculoskeletal fitness.

Analysis of the associations between physical fitness and socioeconomic and demographic factors suggests that: (a) the proportion of adolescents with poor cardiorespiratory fitness was greater among males than among females; (b) that the prevalence of cardiorespiratory unfitness is greater among older male adolescents (aged 17 to 19 years) than among younger male adolescents; (c) that the proportion of adolescents with unhealthy body composition is greater among females and among those nearing the end of their school education; (d) and that more students from middle socioeconomic status families than from lower socioeconomic status families are classed as having musculoskeletal unfitness.

It is therefore recommended that adolescents be encouraged to take part in moderate to vigorous intensity physical activities and sports in order to improve their aerobic capacity and increase their physical strength.

\section{Acknowledgments}

The authors of this study wish to thank the CAPES Foundation for their support (grant BEX 0951/10-2).

\section{References}

1. Erikssen G., Physical fitness and changes in mortality: the survival of the fittest. Sports Med, 2001, 31 (8), 571-576.

2. Hallal P.C., Victora C.G., Azevedo M.R., Wells J.C., Adolescent physical activity and health: a systematic review. Sports Med, 2006, 36 (12), 1019-1030.

3. Silva D.A.S., Lima J.O., Silva R.J.S., Prado R.L., Physical activity level and sedentary behavior among students [in Portuguese]. Rev Bras Cineantropom Desempenho Hum, 2009, 11 (3), 299-306.

4. Ceschini F.L., Andrade D.R., Oliveira L.C., Araújo Júnior J.F., Matsudo V.K.R., Prevalence of physical inactivity and associated factors among high school students from state's public schools. J Pediatr (Rio J), 2009, 85 (4), 301-306, doi: 10.1590/S0021-75572009000400006.

5. Pelegrini A., Petroski E.L., Physical inactivity and its association with nutritional status, body image dissatisfaction and sedentary behavior in adolescents of public schools. Rev Paul Pediatr, 2009, 27 (4), 366-373, doi: 10.1590/ S0103-05822009000400004.

6. Pate R.R., The evolving definition of physical fitness. Quest, 1988, 40 (3), 174-179.

7. Lunardi C.C., Petroski E.L., Body mass index, waist circumference and skinfolds for predicting lipid abnormalities in 11 years old children [in Portuguese]. Arq Bras Endocrinol Metab, 2008, 52 (6),1009-1014, doi: 10.1590/ S0004-27302008000600012.
8. Lopes V.P., Maia J.A.R., Silva R.G., Seabra A., Morais F.P., Health related physical fitness of school children (6 to 10 years) from Azores Islands, Portugal [in Portuguese]. Rev Bras Cineantropom Desempenho Hum, 2004, 6 (2), 7-16.

9. Santos D.M.V., Chaves R.N., Souza M.C., Seabra A., Garganta R., Maia J.A.R., Passing rates on physical fitness. Effects of age, gender, physical activity, overweight and obesity [in Portuguese]. Rev Bras Cineantropom Desempenho Hum, 2010, 12 (5), 309-315, doi: 10.5007/19800037.2010v12n5p309.

10. Luguetti C.N., Ré A.H.N., Böhme M.T.S., Indicators of physical fitness in school children from the Midwest region of São Paulo City [in Portuguese]. Rev Bras Cineantropom Desempenho Hum, 2010, 12 (5), 331-337, doi: 10.5007/1980-0037.2010v12n5p331.

11. Seabra A.F.T., Mendonça D.M.M.V., Garganta R.M., Maia J.A.R., Influence of demographic-biological and social-cultural determinants in the levels of physical activity in children and youngsters [in Portuguese]. Rev Bras Cineantropom Desempenho Hum, 2004, 6 (2), 62-72.

12. Dumith S.C., Azevedo Junior M.R., Rombaldi A.J., Healthrelated physical fitness in students from elementary schools of Rio Grande do Sul, Brazil [in Portuguese]. Rev Bras Med Esporte, 2008, 14 (5), 454-459, doi: 10.1590/S151786922008000500011.

13. Malina R.M., Physical fitness of children and adolescents in the United States: status and secular change. Med Sport Sci, 2007, 50, 67-90.

14. World Health Organization. The Second Decade: Improving adolescent health and development. WHO, Geneve 2001.

15. Brazilian Association of Research Companies, Brazil economic classification criterion [in Portuguese]. 2003. Available at: http://www.abep.org/codigosguias/ ABEP_ CCEB.pdf [accessed: December 10, 2004].

16. Canadian Society for Exercise Physiology. The canadian physical activity, fitness and lifestyle appraisal: CSEP's guide to health active living. $2^{\text {nd }}$ ed. Canadian Society for Exercise Physiology, Ottawa 2003.

17. Glaner M.F., Health-related physical fitness of rural and urban adolescents in relation to the reference criteria [in Portuguese]. Rev Bras Educ Fis Esp, 2005, 19 (1), 13-24.

18. Eisenmann J.C., Welk G.J., Ihmels M., Dollman J., Fatness, fitness, and cardiovascular disease risk factors in children and adolescents. Med Sci Sports Exerc, 2007, 39(8),1251-1256, doi:10.1249/MSS.0b013e318064c8b0.

19. Corseuil H.X., Barbosa D.B.M., Mendes J.K.F., Farias Júnior F.C., Lopes A.S., Petroski E.L., Overweight and blood pressure in adolescents from João Pessoa-PB [in Portuguese]. Rev Educ Fís/UEM, 2009, 20 (2), 273-280, doi: 10.4025/reveducfis.v20i2.4385.

20. Farias E.S., Paula F., Carvalho W.R.G., Gonçalves E.M., Baldin A.D., Guerra-Júnior G., Influence of programmed physical activity on body composition among adolescent students. J Pediatr (Rio J), 2009, 85 (1), 28-34, doi: 10.1590/S0021-75572009000100006.

21. Mikkelsson L.O., Nupponen H., Kaprio J., Kautiainen H., Mikkelsson M., Kujala U. M., Adolescent flexibility, endurance strength, and physical activity as predictors of adult tension neck, low back pain, and knee injury: a 25 year follow up study. Br J Sports Med, 2006, 40, 107-113, doi: 10.1136/bjsm.2004.017350. 


\section{HUMAN MOVEMENT}

E.L. Petroski et al., Health and physical fitness in adolescents

22. Ronque E.R., Cyrino E.S., Dórea V., Serassuelo Júnior H., Galdi E.H.G., Arruda M., Physical fitness diagnosis in schoolchildren of upper socioeconomic status: evaluation for health criterion reference. Rev Bras Med Esporte, 2007,13,71-76, doi:10.1590/S1517-86922007000200001.

23. Serassuelo Júnior H., Rodrigues A.R., Cyrino E.S., Ronque E.R., Oliveira S.R.S., Simões A.C., Health-related fitness in school children of low economic status in the city of Cambé-Paraná [in Portuguese]. Rev Educ Fís/ UEM, 2005, 16 (1), 5-11.

24. Carrel A.L., Clark R.R., Peterson S.E., Nemeth B.A., Sullivan J., Allen D.B., Improvement of fitness, body composition, and insulin sensitivity in overweight children in a school-based exercise program: a randomized, controlled study. Arch Pediatr Adolesc Med, 2005, 159 (10), 963-968.

25. Silva D.A.S., Pelegrini A., Silva J.M.F.L., Petroski E.L., Epidemiology of Abdominal Obesity among Adolescents from a Brazilian State Capital. J Korean Med Sci, 2011, 26 (1), 78-85, doi: 10.3346/jkms.2011.26.1.78.

26. Vasques D.G., Silva K.S., Lopes A.S., Cardiorespiratory fitness of adolescents from Florianópolis, SC [in Portuguese]. Rev Bras Med Esporte, 2007, 13, 376-380, doi: 10.1590/S1517-86922007000600004.
27. Pelegrini A., Silva D.A.S., Petroski E.L., Glaner M.F., Health-related physical fitness in brasilian schoolchildren: data from the Brazil sport program. Rev Bras Med Esporte, 2011, 17 (2), 92-96, doi: 10.1590/S1517-86922011 000200004.

28. Malina R.M., Physical fitness of children and adolescents in the united states: status and secular change. Med Sport Sci, 2007, 50,67-90.

Paper received by the Editors: August 30, 2011

Paper accepted for publication: December 5, 2011

\section{Correspondence address}

Edio Luiz Petroski

Universidade Federal de Santa Catarina

Campus Universitário - Trindade,

Caixa Postal 476

Núcleo de Pesquisa em Cineantropometria e Desempenho Humano

CEP 88,040-900. Florianópolis, SC, Brazil e-mail: petroski@cds.ufsc.br 